cognitive ability. So far, only two researches assess the effect of tDCS in DLB. In this pilot study, we investigate the effects of tDCS in DLB.

Method: Using a double-blind, randomized, sham- controlled and crossover trial design, 11 DLB aged $55-90$ years (mean age 77.8 ) were included in the study. DLB diagnostics is according to DSM-5 criteria. The CDR ratings of DLB participants ranged from 0.5 to 2 . The active tDCS (or sham) process includes consecutive daily sessions of active tDCS (or sham) for 10 days. The anodal electrode was over the left dorsal lateral prefrontal cortex (DLPFC) and the cathodal electrode on the right supraorbital area. In each session, we applied a current intensity of $2 \mathrm{~mA}$ and an electrode size of $25 \mathrm{~cm}^{2}$ for $30 \mathrm{~min}$ in the active group. All subjects received a series of neuropsychological tests, which included CDR, MMSE, CASI, NPI and WCST, before and after these treatment sessions. Chi-square tests, Wilcoxon signed rank tests and Mann-Whitney $U$ tests were used to assess the differences in participant demographic characteristics and to compare the differences among groups.

Results: On CASI, MMSE, NPI and WCST, there were no statistically significant differences between preand post the 10-session course for the active and the sham groups. No side effects reported during or immediately after active tDCS stimulation.

Conclusion: These results suggest that left DLPFC anodal, and right deltoid cathodal tDCS, do not improve cognition, behavioral and psychological symptoms in DLB. Larger-scale trials are needed to confirm the effect of tDCS in DLB.

Key words: Transcranial Direct Current Stimulation, Dementia with Lewy Bodies, cognitive function, Wisconsin Card Sorting Test

\title{
420 - Ketamine treatment in geriatric depression
}

Authors: Janette Leal, MD; Maria Lapid, MD; Simon Kung, MD

Treatment resistant depression can be very disabling and has a significant negative impact on a patient, their family or caregivers, and the society. There is a growing evidence on the efficacy and safety of ketamine for treatment resistant depression. Ketamine is a racemate consisting of esketamine and arketamine, is an $\mathrm{N}$-methyl $\mathrm{D}$-aspartate receptor antagonist and comes in different formulations and in fact intranasal ketamine is FDA-approved in the US. Despite the existing evidence and FDA approval for treatment resistant depression, data on older individuals remains limited. Late life depression especially those that are treatment resistant can be very disabling, with significant functional and cognitive impairments, increased morbidity and mortality, and the psychosocial burdens.

This presentation describes outcomes for 2 cases of older individuals with treatment resistant depression who were treated with intranasal ketamine. One improved, and one did not. The scarce available literature of the use of ketamine in the geriatric population shows ketamine is well tolerated and effective. Remission rates in 3 separate show remission rates of $46.5 \%, 57 \%$ and $69.5 \%$ respectively. The discussion will include a review of the mechanism of action of ketamine as a novel antidepressant, the mixed evidence for its role in treatment resistant late life depression, and the practical and operational aspects relevant to running a ketamine clinic. 